\title{
Microfluidic interactions between red blood cells and drug carriers by image analysis techniques
}

\author{
Rosa D’APOLITO ${ }^{1,5}$, Francesca TARABALLI ${ }^{2}$, Silvia MINARDI ${ }^{3}$, Xuewu LIU $^{2}$, Sergio CASERTA ${ }^{1,5}$, \\ Armando CEVENINI ${ }^{4}$, Ennio TASCIOTTI ${ }^{2}$, Giovanna TOMAIUOLO ${ }^{1,5 *}$, Stefano GUIDO ${ }^{1,5}$ \\ * Corresponding author: Tel.: ++39 081 7682261; Fax: ++39 081 2391800; Email: g.tomaiuolo@unina.it \\ 1 Dipartimento di Ingegneria Chimica, dei Materiali e della Produzione Industriale, Università di Napoli \\ Federico II, Italy \\ 2 Department of NanoMedicine, The Methodist Hospital Research Institute, Houston, TX, USA \\ 3 National Research Council of Italy - Institute of Science and Techonology for Ceramics \\ 4 Department of Molecular Medicine and Medical Biotechnology, Università di Napoli Federico II, Italy \\ 5 CEINGE Biotecnologie avanzate, Napoli, Italy
}

\begin{abstract}
Blood is a complex biological fluid composed of deformable cells and platelets suspended in plasma, a protein-rich liquid. The peculiar nature of blood needs to be considered when designing a drug delivery strategy based on systemically administered carriers. Here, we report on an in vitro fluid dynamic investigation of the influence of the microcapillary flow of red blood cells (RBCs) on micron sized carriers by high speed imaging methods. The experiments were carried out in a $50 \mu \mathrm{m}$ diameter glass capillary that mimicked the hydrodynamic conditions of human microcirculation. Spherical $\mu$-particles ( $\mu$-Ps), with sizes ranging between 0.5 and $3 \mu \mathrm{m}$, were tested. Images of the flowing RBCs and $\mu$-Ps were acquired by a highspeed/high-magnification microscopy. The transport and distribution of rigid particles in a suspension of RBCs under shear flow were followed for: i) the migration of RBCs towards the vessel centerline due to their deformability; ii) the cross-flow migration of $\mu$-Ps towards the vessel wall due to their hydrodynamic interactions with RBCs; iii) the radial distribution of $\mu-$ Ps in the presence of RBCs. This study suggests that the therapeutic efficacy of $\mu-P s$ could be ultimately affected by their interactions with the flowing RBCs in the vasculature.
\end{abstract}

Keywords: Red blood cells, $\mu$-particles, Microcirculation, Drug delivery

\section{Introduction}

Human blood is a complex non-Newtonian biological fluid consisting in a suspension of cells in plasma, a protein-rich Newtonian fluid. The blood cells are constituted mainly ( $\sim 99 \%)$ by red blood cells (RBCs), the remaining being white blood cells and platelets. One of the most remarkable properties of $\mathrm{RBCs}$ is their high deformability, that allows the flow even through microcapillaries of diameter smaller than their size (Guido and Tomaiuolo, 2009; Lanotte et al., 2014; Tomaiuolo et al., 2011; Tomaiuolo and Guido, 2011; Tomaiuolo et al., 2012; Tomaiuolo et al., 2009). The peculiar properties of RBCs need to be considered when designing a drug delivery strategy based on systemically administered carriers and the delivery efficiency has to be evaluated, by analyzing the distribution of micro-carriers within blood vessels radius (Zhao et al., 2012). Recently, computational modeling has been used to demonstrate that, while nano-particles (diameter about $100 \mathrm{~nm}$ ) present a uniform radial distribution and limited near-wall accumulation when flowing with RBCs, micro-particles (diameter about 1 $\mu \mathrm{m})$ tend to accumulate near the tube wall (Lee et al., 2013; Saadatmand et al., 2011), as happens for platelets when they concentrate in the cell-free layer near the vessel wall, in a mechanism known as margination (Aarts et al., 1988). The latter is a combination of three phenomena: i) the migration of $\mathrm{RBC}$ towards the vessel centerline due to their deformability, leaving a cell-free layer near the vessel wall (Geislinger and Franke; Lee et al., 2013; Zhao et al., 2012); ii) the concentration of platelets 
in the cell-free layer near the wall, due to cell rigidity, that allows a rigid-body flipping motion near the wall (Zhao et al., 2012); iii) the cross-flow migration of platelets towards the vessel wall due to their hydrodynamic interactions with RBCs (Aarts et al., 1988; Zhao et al., 2012). In vitro experiments evidenced that the presence of deformable RBCs is fundamental for the near wall concentration of platelets (Aarts et al., 1988; Zhao et al., 2007).

Here, we report on an in vitro flow-based imaging method to investigate the fluid dynamic influence of RBCs on micron sized drug carriers migration in the cell-free layer near the vessel wall, taking inspiration by platelets margination mechanism. A suspension of poly D,L-lactic-CO-glycolic acid (PLGA) micro-particles ( $\mu$-Ps) and RBCs has been used. PLGA is a copolymer of poly lactic acid (PLA) and poly glycolic acid (PGA). The physical properties of the polymer depend upon multiple factors, including the initial molecular weight, the relative ratio of the polymeric monomers, the exposure to water (surface shape) and storage temperature (Houchin and Topp, 2009). For its suitability and long clinical experience, PLGA, can be considered the most popular biodegradable polymer. Various polymeric devices like microspheres, microcapsules, nanoparticles, pellets, implants and films have been fabricated using these polymer (Cheng et al., 2007; Esmaeili et al., 2008; Fan et al., 2012; Houchin and Topp, 2009; Li et al., 2001; Makadia and Siegel, 2011; Wischke and Schwendeman, 2008). PLGA has been mostly formulated into delivery systems for vehicle different drug classes, such as vaccines, peptides, proteins and micro-molecules (Li et al., 2001). Although many numerical works on particles radial distribution in tube flow (Bhattacharya et al., 2013; Lan and Khismatullin, 2012; Tan et al., 2012; Zhao et al., 2012) are present in literature, to our knowledge only few experimental works (Saadatmand et al., 2011; Tan et al., 2012) focused on this issue. The aim of the present study is to provide a quantitative fluiddynamic analysis of the influence of RBCs flow on micro-drug carriers migration, to model in vitro the mechanisms that regulates the transport of injectable carriers in microcirculation and to help the design of micro-particles with physical and chemical features optimized for vascular delivery.

\section{Materials and methods}

\subsection{Blood sample}

Fresh venous blood samples were drawn from healthy donors and diluted to a concentration of $10 \%$ by volume with ACD anticoagulant $\quad(0.6 \%$ citric acid, $1.1 \%$ anhydrous dextrose, $2.3 \%$ sodium citrate, $96 \%$ water) and bovine serum albumin BSA. All the samples were used within $4 \mathrm{~h}$ from collection. This concentration has been selected in order to have the typical hematocrit Hct value (i.e., the percentage of cell volume with respect to total blood volume) found in microcirculation, where Hct ranges from 10 to 26\% (Fung, 1997). The higher values of Hct (around 45\%) measured in healthy blood tests are associated with large vessels, whereas in going from the macro- to the microvasculature Hct decreases due to entrance effects and the inhomogeneous radial distribution of $\mathrm{RBCs}$, which tend to concentrate along the centerline (the so called Fahraeus-Lindqvist effect (Albrecht et al., 1979).

\subsection{PLGA $\mu$-particles}

The classical methods for preparation of PLGA particles are bottom-up techniques such as phase separation, spray drying or solvent extraction/evaporation-based processes. The preparation technique can vary depending on the application of the PLGA vehicles (Bouissou et al., 2006; Chaisri et al., 2009; Rosca et al., 2004). In the present work, PLGA (50:50) microspheres (3-0.5 $\mu \mathrm{m})$ have been prepared by double emulsion method. This method has been applied because is the most used to encapsulate hydrophilic payload such as proteins, peptides and nucleotides (Ruhe et al., 2003). The double emulsion method involves the formation of a primary water-in-oil emulsion (W1/O), which is then dispersed into a secondary aqueous solution 
(W2) to form a (W1/O/W2) emulsion. After the evaporation/extraction of the solvents, porous microspheres are formed with size depending on different parameter such as polymer concentration, evaporation velocity, and used surfactant (Makadia and Siegel, 2011).

Synthesis. PLGA microspheres (PLGA ester terminated (lactide/glycolide $=50: 50$ ) viscosity range 0.95-1.20 dl from LACTEL (Pelham, Alabama, USA)) were prepared by a modified $\mathrm{S} / \mathrm{O} / \mathrm{W}$ emulsion method as mentioned in our previous studies (Ruhe et al., 2003). Briefly, PLGA (50:50) was dissolved in dichloromethane (DCM) to form $20 \%$ or $5 \%$ w/v PLGA/DCM solution. The organic phase containing the drug was mixed with of PVA, $2.5 \% \mathrm{w} / \mathrm{v}$ by vortex mixing and sonication (3000 rpm) for 3 minutes. The mixture was gradually dropped into a solution of PVA1\% $\mathrm{w} / \mathrm{v}$. The resulting suspension was stirred with a magnetic stir bar for $6 \mathrm{~h}$ and the DCM was eliminated by evaporation. The suspension was washed 3 times by centrifugation at 5000 rpm for 10 minutes. The microspheres were filtered by MF-Millipore ${ }^{\mathrm{TM}}$ Membrane Filters at $3 \mathrm{um}$ and then freeze-dried and stored as well at $-80^{\circ} \mathrm{C}$. After the double emulsion synthesis (Figure $1 \mathrm{~A}$ ) the mean diameter of the PLGA composite microparticles was $2.1 \mu \mathrm{m}(\mathrm{SD} \pm 1.3)$. Multiple syntheses of microparticles have been performed to demonstrated repeatable distributions. Scanning electron microscopy (SEM) images of the microparticles showed a regular spherical morphology. The apparent size of the microparticles has been confirmed by SEM images (Figure $1 \mathrm{~B}$ ). Table 1 showed the zeta potential value of the particles used in this study. The values reported, around $-25 \mathrm{mV}$, are similar to those reported in literature (Pawar et al., 2010).

Zeta potential. The zeta potential of the PLGA particles was analyze using a Zetasizer ZEN3600 (Malvern, Worcestershire, UK). 2 $\mathrm{ml}$ of particle suspension were injected into a sample cell countering filled with phosphate buffer (PB, 1.4Ml, pH 7.3). The cell was sonicated for 2 minutes, and then an electrode- probe was put into cell. Measurements were performed at RT in triplicates.

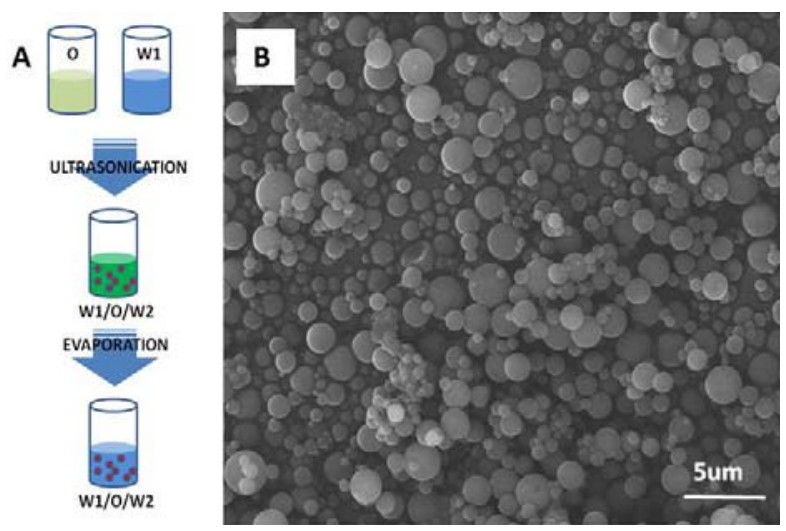

Fig. 1. Synthesis and Characterization of PLGA microparticles. A) Scheme of the double emulsion synthesis. B) SEM images of PLGA microparticles.

Table I: Values of Zeta potential of the microparticles.

\begin{tabular}{c|c|c}
\hline Sample name & Size & Zeta potential \\
\hline PLGA $(50: 50) 5 \% \mathrm{w} / \mathrm{w}$ & $0.5-3 \mu \mathrm{m}$ & $-27.2 \mathrm{mV} \pm 0,65 \mathrm{mV}$ \\
\hline $\mathrm{PLGA}(50: 50) 20 \% \mathrm{w} / \mathrm{w}$ & $0.5-3 \mu \mathrm{m}$ & $-25.6 \mathrm{mV} \pm 0,75 \mathrm{mV}$ \\
\hline
\end{tabular}

Size distribution. The particle size distribution and count was obtained using a Z2 Coulture Particles Counter and Size Analyzer (Beckman Coulter, Fullerton, CA). $10 \mu \mathrm{l}$ of the samples were dispersed in the balanced electrolyte solution (ISOTON II Diluent, Beckman Coulter Fullerton, CA0) and sonicated for $5 \mathrm{~s}$ to ensure a homogenous dispersion.

Scanning electron Microscope (SEM). Samples were washed twice in deionized water to remove salt, placed on aluminum mounts (Ted Pella; Redding, CA), and left in a vacuum desiccator to dry overnight. Samples were analyzed in a Zeiss Neon 40 microscope equipped with an in-lens detector at an acceleration voltage between 5 and $10 \mathrm{keV}$ at a working distance of approximately $5 \mathrm{~mm}$.

\section{$2.3 \mu$-Ps and RBCs/ $\mu$-Ps suspensions}

Two different suspensions have been used: a $\mu$-Ps suspension, used as control, made by poly (lactide-co-glycolide) PLGA $\mu$-Ps (Figure 1) suspended in ACD and BSA $(0.004 \%$ weight) and a $\mu$-Ps/RBCs suspension consisting of $\mu$-Ps $(0.004 \%$ weight $)$ in a RBCs suspension with $\mathrm{Hct}=10 \%$. 


\subsection{Experimental apparatus}

Images of the flowing suspensions are acquired by a high speed video camera (Phantom 4.3, operated up to 1000 frames/s) and by using a high magnification oil immersion objective $(100 \times)$. The experiments are carried out in $50 \mu \mathrm{m}$ diameter silica microcapillary (Polymicro Technologies) placed on the motorized x-y stage (Ludl) of an inverted microscope (Zeiss Axiovert 100) equipped with a motor assembly for remote focus control (Ludl). The capillary tube is connected to a micro-pumping system, in which the $\mu$-Ps/RBCs suspensions have been loaded (Figure 2). The $50 \mu \mathrm{m}$ silica capillary mimics the hydrodynamic conditions of human microcirculation, such as laminar flow and physiological pressure $(20-60 \mathrm{mmHg})$ (Whitmore, 1967).

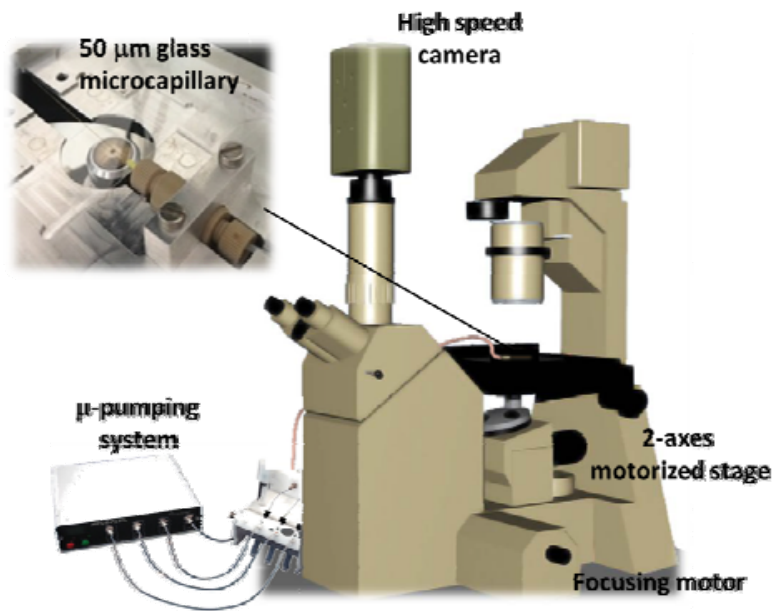

Fig. 2. Schematic of the experimental apparatus, and zoom of the $50 \mu \mathrm{m}$ glass microcapillary

All the measurements were performed in the center plane of $50 \mu \mathrm{m}$ glass capillary (length 5,5 cm) (Figure 3), at different pressure drops (20 and $55 \mathrm{mmHg}$ ).

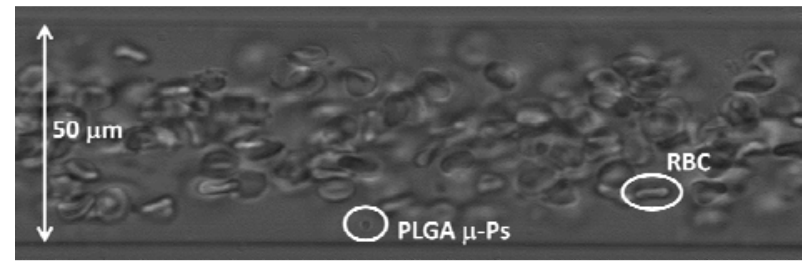

Fig. 3. $\mu-\mathrm{Ps} / \mathrm{RBCs}$ suspension flow in a $50 \mu \mathrm{m}$ glass microcapillary.

\section{Results}

The transport and distribution of microparticles in a suspension of deformable RBC under Poiseuille flow are related to: i) the velocity profile of both $\mu$-Ps and RBCs in the capillary; ii) the migration of RBC towards the vessel centerline due to their deformability, leaving a cell-free layer near the vessel wall; iii) the cross-flow migration of micro-particles towards the vessel wall due to their hydrodynamic interactions with RBCs; iv) the radial distribution of $\mu$-particles in the presence of RBCs. To explore the effect of RBCs on $\mu$-Ps flow and margination, the velocity profiles and the radial distribution of $\mu$-Ps of both the suspensions ( $\mu$-Ps and $\mu$ Ps/RBCs) have been determined by using high-speed video microscopy and image analysis.

\subsection{Velocity profiles}

The velocity profiles have been determined by using image analysis, by tracking both $\mu$-Ps and RBCs along the capillary. $\mu$-Ps velocity along radius-direction is shown in Figure 4.
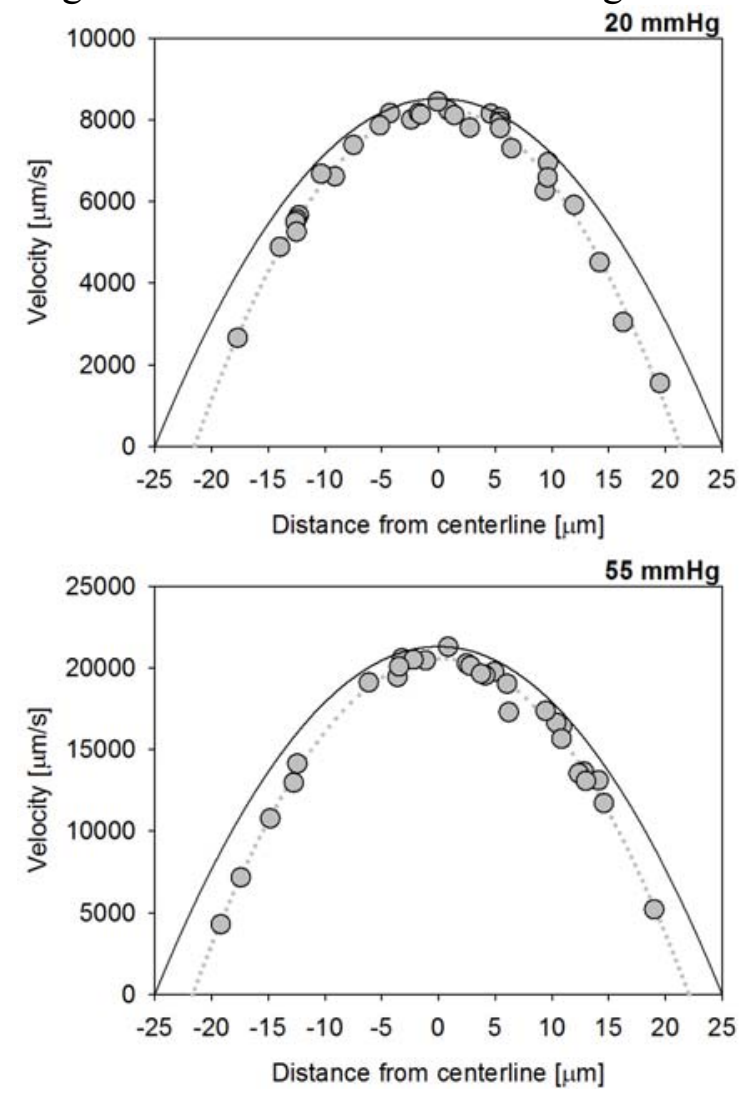

Fig. 4. $\mu$-Ps velocity profile at $20,55 \mathrm{mmHg}$

The dots represent the $\mu$-Ps data at two different pressure drops (20 and $55 \mathrm{mmHg}$ ) and the continuous line represents the fluid velocity as calculated from Poiseuille tube 
flow, at the same pressure drops.

The imposed pressure drop is taken as being equal to the pressure drop in the microcapillary, the other pressure losses being negligible.

We can observe a not perfect correspondence between the fluid velocity (continuous line) and the experimental data. This is the consequence of a well-known optical artifact resulting from the difference in refractive indices of glass $\left(\mathrm{N}_{\mathrm{g}} \sim 1,5\right)$ and suspending liquid $\left(\mathrm{N}_{\mathrm{sl}} \sim 1,33\right)$. In order to correct this artifact, it was made a curvature correction by using the Snell's law (Smith et al., 2003).

In Figure 5 it can be seen that experimental data after the curvature correction (dark grey circles) are in good agreement with the fluid velocity as calculated by Poiseuille's law (continuous line). In fact, $\mu$-Ps show the same velocity of the fluid field. All the following figures will be shown in the curvaturecorrected form.

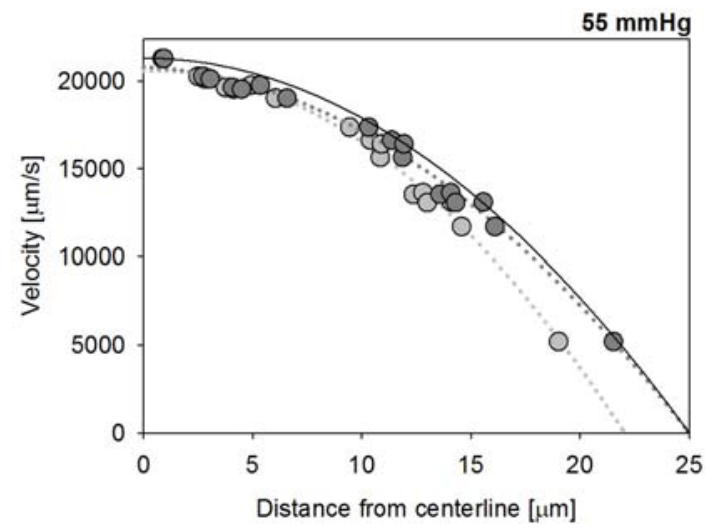

Fig. 5. Curvature correction at $55 \mathrm{mmHg}$

In Figure 6, the plot of $\mu$-Ps (grey circles) and RBCs (dark triangles) velocity profiles as a function of the capillary radius is shown for two different values of pressure drops. The continuous line is the average velocity of the suspending fluid as calculated for Poiseuille flow. RBC velocity stays in between the average and maximum velocity of the suspending fluid, the latter being twice the former in Poiseuille tube flow. Thus, RBC velocity is lower than the velocity of the suspending fluid in absence of RBCs (continuous line). Similar results are found for other deformable objects, such as liquid vesicles (Misbah, 2012; Pommella et al., 2013) and capsules (Bagchi and Kalluri, 2009; Misbah, 2012), which are considered as model systems of actual RBCs. It can be noticed that $\mu$-Ps velocity in presence of RBCs is lower than velocity of the solution made by $\mu$-Ps only (Fig. 4) and close to the one of RBCs, indicating that the presence of RBCs affects the flow behavior of the $\mu$-Ps. In other words, the velocity field is dominated by the RBCs.
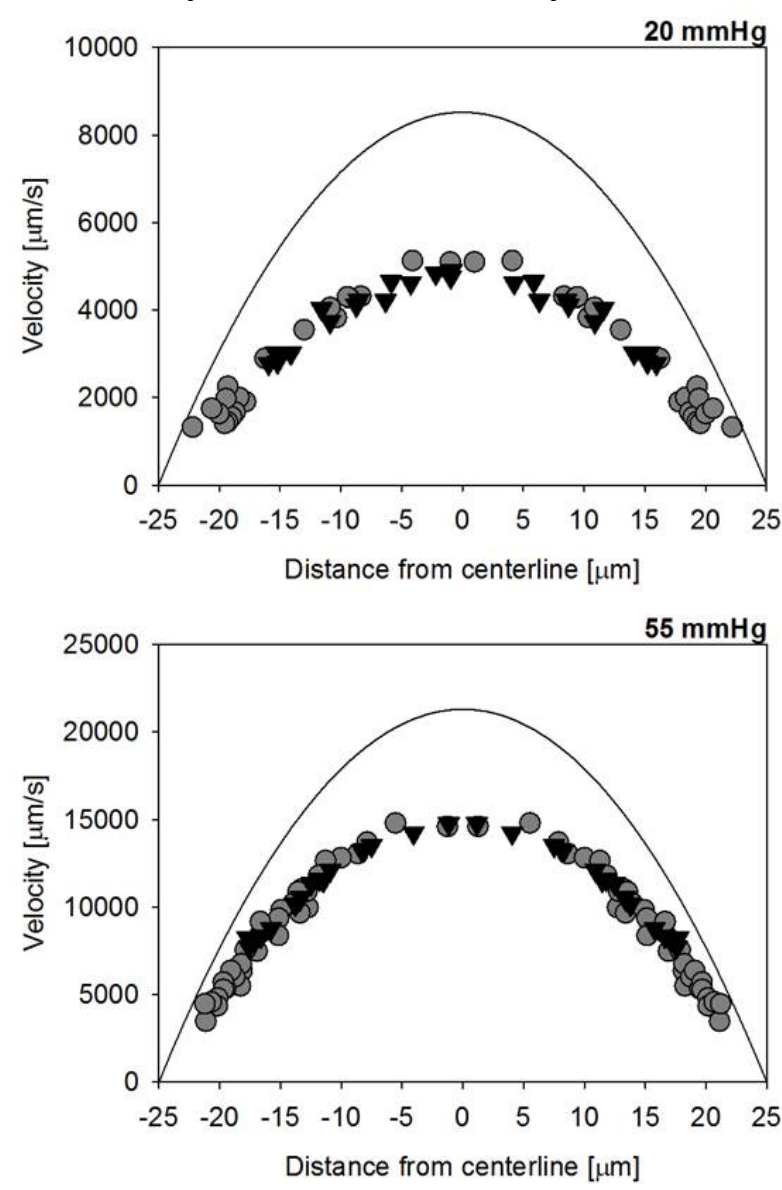

Fig. 6. $\mu$-Ps/RBCs velocity profile at 20 and $55 \mathrm{mmHg}$

\subsection{Radial distribution of $\mu$-Ps}

The radial distributions of $\mu$-Ps have been determined by using image analysis, by tracking $\mu$-Ps along the capillary radius.

In order to quantify the radial distribution of the $\mu$-Ps, the center plane of the capillary was divided in 10 regions, each accounting for $10 \%$ of the capillary section. The number of $\mu$ Ps present in each layer was divided by the total number of particles. The so obtained normalized mean concentration of $\mu$-Ps with (black bars) and without (dark grey bars) RBCs was plotted as a function of capillary radius in Figure 7. In absence of RBCs, there 
is a uniform distribution of $\mu$-Ps along the capillary radius, except for the layers close to the wall (from -25 to $-20 \mu \mathrm{m}$ and from 20 to $25 \mu \mathrm{m}$ ), due to the wall-effect (Geislinger and Franke). In the absence of RBCs, no matter how much the pressure drop increases, the $\mu$ Ps distribution almost keeps un-changed. Such non uniform $\mu$-Ps distribution is induced by the RBCs axial migration (Albrecht et al., 1979).
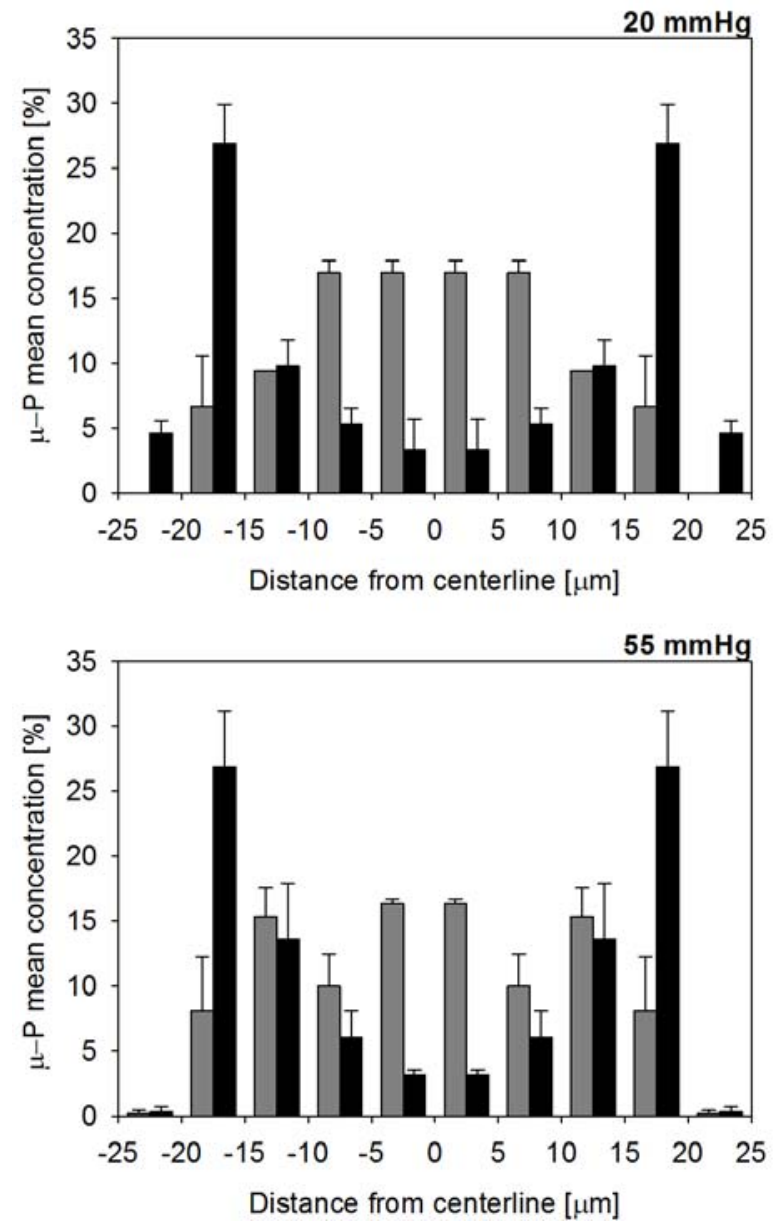

Fig. $7 \mu$-Ps distribution profile across the capillary with (black) and without (grey) RBCs at 20 and $55 \mathrm{mmHg}$.

The percentage of $\mu$-Ps (called marginated $\mu$-Ps) in the outer layers (from -25 to $-15 \mu \mathrm{m}$ and from 15 to $25 \mu \mathrm{m}$ ) with (black bars) and without (dark grey bars) RBCs was plotted as a function of pressure drop in Figure 8 .

It is shown that the number of $\mu$-Ps in presence of RBCs increases in the outer layers from $\sim 16 \%$ (i.e., $\sim 8 \%$ per each outer layer) to $\sim 60 \%$ (i.e., $\sim 30 \%$ per each outer layer) compared to that in absence of RBCs.

Similar increase of particle concentration near-wall has been reported for platelets and white blood cells distribution in capillary flow (Crowl and Fogelson, 2010; Nobis et al., 1985).

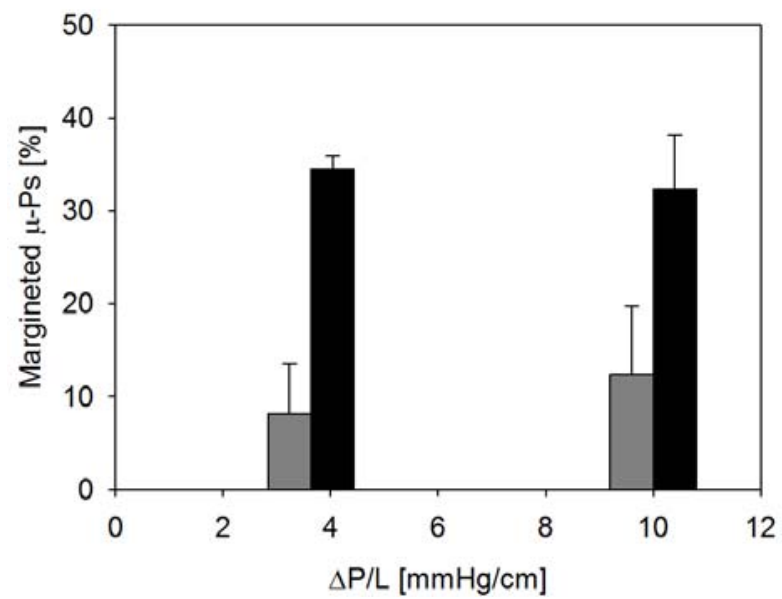

Fig. 8 Marginated $\mu$-Ps with (black) and without (grey) $\mathrm{RBCs}$ at 20 and $55 \mathrm{mmHg}$.

Moreover, as the pressure drop increases, the $\mu$-Ps mean concentration near the wall decreases, due to the increase of the cell freelayer with pressure drop (Albrecht et al., 1979).

\section{Conclusions}

The aim of this work is to elucidate the mechanisms that regulates the transport of injectable carriers in microcirculation and to help the design of micro-particles with physical and chemical features optimized for vascular delivery. We observed that in the absence of RBCs the $\mu$-Ps showed a uniform radial distribution, moving with the same velocity of the fluid field. In presence of $\mathrm{RBCs}$, instead, the particles located near or at the centerline drifted laterally, moving towards the wall and accumulated in the cell-free layer, in a pressure drop dependent manner.

This study suggests that the therapeutic efficacy of $\mu$-particles could be ultimately affected by their interactions with circulating cells and by their behavior in the vasculature. This study suggests that the therapeutic efficacy of micro-carriers could be ultimately affected by their interactions with circulating cells and by their behavior in the microvasculature. 


\section{References}

Aarts, P., et al., 1988. Blood-platelets are concentrated near the wall and red blood-cells, in the center in flowing blood. Arteriosclerosis. 8, 819-824.

Albrecht, K., et al., 1979. The Fahraeus effect in narrow capillaries (i.d. 3.3 to 11.0 micron). Microvasc Res. 18, 33-47.

Bagchi, P., Kalluri, R. M., 2009. Dynamics of nonspherical capsules in shear flow. Physical Review E. 80.

Bhattacharya, S., et al., 2013. Radial distribution and axial dispersion of suspended particles inside a narrow cylinder due to mildly inertial flow. Physics of Fluids. 25.

Bouissou, C., et al., 2006. The influence of surfactant on PLGA microsphere glass transition and water sorption: Remodeling the surface morphology to attenuate the burst release. Pharmaceutical Research. 23, 1295 1305.

Chaisri, W., et al., 2009. Preparation and Characterization of Cephalexin Loaded PLGA Microspheres. Current Drug Delivery. 6, 69-75.

Cheng, J., et al., 2007. Formulation of functionalized PLGA-PEG nanoparticles for in vivo targeted drug delivery. Biomaterials. 28, 869-876.

Crowl, L. M., Fogelson, A. L., 2010. Computational model of whole blood exhibiting lateral platelet motion induced by red blood cells. International Journal for Numerical Methods in Biomedical Engineering. 26, 471-487.

Esmaeili, F., et al., 2008. PLGA nanoparticles of different surface properties: Preparation and evaluation of their body distribution. International Journal of Pharmaceutics. 349, 249-255.

Fan, D., et al., 2012. Mesoporous SiliconPLGA Composite Microspheres for the Double Controlled Release of Biomolecules for Orthopedic Tissue Engineering. Advanced Functional Materials. 22, 282-293.
Fung, Y.-c., 1997. Biomechanics: circulation. Springer.

Geislinger, T. M., Franke, T., Hydrodynamic lift of vesicles and red blood cells in flow — from Fåhræus \&amp; Lindqvist to microfluidic cell sorting. Advances in Colloid and Interface Science.

Guido, S., Tomaiuolo, G., 2009. Microconfined flow behavior of red blood cells in vitro. C. R. Physique. 10, 751-763.

Houchin, M. L., Topp, E. M., 2009. Physical Properties of PLGA Films During Polymer Degradation. Journal of Applied Polymer Science. 114, 28482854.

Lan, H., Khismatullin, D. B., 2012. A numerical study of the lateral migration and deformation of drops and leukocytes in a rectangular microchannel. International Journal of Multiphase Flow. 47, 73-84.

Lanotte, L., et al., 2014. Red blood cell dynamics in polymer brush-coated microcapillaries: A model of endothelial glycocalyx in vitro. Biomicrofluidics. 8, -.

Lee, T.-R., et al., 2013. On the near-wall accumulation of injectable particles in the microcirculation: smaller is not better. Scientific Reports. 3.

Li, Y. P., et al., 2001. PEGylated PLGA nanoparticles as protein carriers: synthesis, preparation and biodistribution in rats. Journal of Controlled Release. 71, 203-211.

Makadia, H. K., Siegel, S. J., 2011. Poly Lactic-co-Glycolic Acid (PLGA) as Biodegradable Controlled Drug Delivery Carrier. Polymers. 3, 13771397.

Misbah, C., 2012. Vesicles, capsules and red blood cells under flow. Journal of Physics: Conference Series. 392, 012005.

Nobis, U., et al., 1985. Radial distribution of white cells during blood flow in small tubes. Microvascular research. 29, 295304. 
Pawar, D., et al., 2010. Evaluation of Mucoadhesive PLGA Microparticles for Nasal Immunization. Aaps Journal. $12,130-137$.

Pommella, A., et al., 2013. Dynamic flow behaviour of surfactant vesicles under shear flow: role of a multilamellar microstructure. Soft Matter. 9, 75457552.

Rosca, I. D., et al., 2004. Microparticle formation and its mechanism in single and double emulsion solvent evaporation. Journal of Controlled Release. 99, 271-280.

Ruhe, P. Q., et al., 2003. rhBMP-2 release from injectable poly(DL-lactic-coglycolic acid)/calcium-phosphate cement composites. Journal of Bone and Joint Surgery-American Volume. $85 \mathrm{~A}, 75-81$.

Saadatmand, M., et al., 2011. Fluid particle diffusion through high-hematocrit blood flow within a capillary tube. Journal of Biomechanics. 44, 170-175.

Smith, M., et al., 2003. Near-wall micro-PIV reveals a hydrodynamically relevant endothelial surface layer in venules in vivo. Biophys J. 85, 637-45.

Tan, J., et al., 2012. Influence of red blood cells on nanoparticle targeted delivery in microcirculation. Soft Matter. 8, 1934-1946.

Tomaiuolo, G., et al., 2011. Microfluidics analysis of red blood cell membrane viscoelasticity. Lab Chip. 11, 449-54.

Tomaiuolo, G., Guido, S., 2011. Start-up shape dynamics of red blood cells in microcapillary flow. Microvasc Res. $82,35-41$.

Tomaiuolo, G., et al., 2012. Comparison of two flow-based imaging methods to measure individual red blood cell area and volume. Cytom Part A. 81.

Tomaiuolo, G., et al., 2009. Red blood cell deformability in microconfined shear flow. Soft Matter. 5, 3736-3740.

Whitmore, R. L., 1967. The flow behaviour of blood in the circulation. Nature. 215, 123-6.

Wischke, C., Schwendeman, S. P., 2008.
Principles of encapsulating hydrophobic drugs in PLA/PLGA microparticles. International Journal of Pharmaceutics. 364, 298-327.

Zhao, H., et al., 2012. Shear-induced particle migration and margination in a cellular suspension. Physics of Fluids. 24, 011902-21.

Zhao, R., et al., 2007. Investigation of platelet margination phenomena at elevated shear stress. Biorheology. 44, 161-177. 